\title{
Overview of Some R\&D at the University of Applied Sciences Wädenswil
}

\author{
Marco Bachmann*
}

Abstract: An overview is given by several authors on various activities in research and development at the University of Applied Sciences Wädenswil.

Keywords: Beverage · Food Technology · Nutrition · Research and Development ·

University of Applied Sciences Wädenswil

\section{Microbial Environmental Monitoring in Food and Packaging Industry}

\section{Corinne Gantenbein-Demarchi University of Applied Sciences Wädenswil}

Recently, much emphasis has been placed by industry on the improvement of air quality in food and packaging plants as a means of enhancing product safety and shelf life. All locations or points at which raw materials, semi- and finished products are exposed to environmental air (not controlled or in clean rooms) represent potential hazardous routes of product contamination by air-borne microorganisms. The European Union Directive 93/43/CEE for food hygiene and respective national regulations stipulate that food manufacturers have to implement internal hygienic management on their own authority. Appropriate measurements within such management systems should be carried out according to analysis of critical points in agreement with HACCP principles and Codex Alimentarius. The purpose of this proposed RTD action would be the definition and laying

\footnotetext{
${ }^{\star}$ Correspondence: Dr. M. Bachmann

University of Applied Sciences

Food Technology Department

P.O Box 335

$\mathrm{CH}-8820$ Wädenswil

Tel.: +4117899900

Fax: +4117899950

E-Mail: m.bachmann@hswzfh.ch
}

down of standards for microbial environmental self-controls in manufacturing plants.

\section{Development of Gluten-free Bakery Products}

Marco Bachmann ${ }^{a}$, Peter Rudin ${ }^{b}$, and Michael Kleinert ${ }^{b}$

aUniversity of Applied Sciences

Wädenswil; 'bäckereifachschule

Richemont Luzern

Coeliac disease is a chronic indigestibility based on a reaction between certain proteins and the mucus of the intestine. The proteins - the gluten - are found in wheat, rye, barley, oat, and spelt. Several years ago it was thought that 1 person in 1000 is affected by this problem. Statistical data of Ireland and Finland show that the frequency is much higher: 1 person in 100 is affected. The reasons for this increase are the improvements in diagnosis. So far only one method for avoiding the indigestibility is known, i.e. not eating any milled products containing these proteins. The Food Technology Department of the University of Applied Sciences Wädenswil and the Bakery School in Luzern have developed in the last three years different recipes for gluten-free bread which showed in comparison with normal bread acceptable crust and crumb and equivalent nutritional value. The new gluten-free puff pastry had the typical puffing and biting characteristics. These new products will help people with coeliac diseases to choose different bread types and other bakery products made with puff pastry.

\section{Treatment of Fluid Foods by Inductive Heating}

\section{Christian Gerhards \\ University of Applied Sciences \\ Wädenswil}

By the inductive heating process a.c. electricity is used to induce a magnetic flux in the food passing through the apparatus. Similar to heat generation by microwaves, heat is generated inside the product by rotation of molecules and vibrations of ions. Thus heat is distributed evenly through the food, avoiding local thermal stress. In addition, solid particles can be heated quickly, as heat transfer into the particles is not the limiting step. In case of a breakdown, the electric current can be immediately switched off, preventing burning of the product.

In recent experiments, inductive heating was applied to various liquid foods. Samples of apple juice, tomato sauce and milk were heated, using the Actijoule process (Actini SA, Evian Les Bains, France). Parameters varied were temperature $\left(80-100{ }^{\circ} \mathrm{C}\right)$ and holding time (30-180 s), resulting in different PE values from 0.35 to 20. Inactivation of microorganisms was measured by plate count before and after experiments. Defined initial number of microorganisms was given by inoculation of S. cerevisiae and penicillium. The quality of the food products after heat treatment was examined by measuring the vitamin $\mathrm{C}$ content, and colour (in the case of fruit juice), $\beta$-lactoglobulin, furosin and peroxidase content, respectively (in the case of milk). 


\section{The Influence of High-energy Short-wave Radiation and Other Environmental Factors on the Genesis of Compounds Affecting the Wine Quality in Vitis Vinifera L., c.v. Müller-Thurgau}

Tilo Hühn ${ }^{a}$, Wolf-Rüdiger Sponholz ${ }^{b}$, Konrad Bernath ${ }^{a}$, Adrian Friedmann ${ }^{c}$, Georg Hess ${ }^{a}$, Hildegard Muno ${ }^{b}$, and Walter Fromm ${ }^{b}$

aUniversity of Applied Sciences

Wädenswil, Department of Beverage

Technology and Oenology,

Wädenswil; ' ${ }^{\mathrm{G}}$ Geisenheim Research

Institute, Department of Micro-

biology and Biochemistry,

Von-Lade-Str. 1, D-65366 Geisen-

heim; 'Novartis Crop Protection AG,

Research Formulation, Basel

A field experiment was established during the growing periods 1997 and 1998 with the variety Müller-Thurgau on an experimental site, which induces slight and severe stress (water and nitrogen) on the grapevine. The vines were protected against certain wavelengths of UV-radiation by an absorbing plastic film (1997) and an UV-absorber (1998) applied to the grape zone. It was found that the content of 2-aminoacetophenone and skatole increased in the wines of the control plots while the indole content increased in the treated plots. The finding indicates that 'environmental stress' can induce the formation of quality-reducing substances in wines. Further work is necessary to elucidate the different physiological processes which are involved in plants and the fermentation process.

\section{Gastroenteritis Outbreaks due to Infections with 'Norwalk-like Viruses' (NLV) in Switzerland}

\section{Rainer Fretz, Paul Svoboda, Thomas Lüthi, and Andreas Baumgartner Liestal, Wädenswil, Bern}

'Norwalk-like viruses' (NLV) are a genus belonging to the family of Caliciviridae. These viruses are mainly transmitted by the faecal-oral route and by aerosols. They are one of the most common causes of outbreaks of nonbacterial gastroenteritis. In the US, NLVs are thought to be responsible for an estimated $67 \%$ of food borne infections and for $96 \%$ of nonbacterial cases of gastroenteritis.

By outbreak investigation in the German-speaking part of Switzerland, an attempt has been made for the first time to assess and describe the epidemic potential of NLVs in our country. In cooperation with different Cantonal Laboratories and public health specialists, outbreaks with NLVs as possible causative agent, were recorded, analysed and classified during the year 2001.

In total, seven NLV outbreaks could be documented. In five cases, NLVs were detected by RT-PCR in stool samples, in one case the PCR product could not be confirmed by sequencing and in another case, no samples were provided. The clinical symptoms were typical and in accordance with the definition of the NLV-infection. The outbreaks occurred in four different settings: four outbreaks concerned different school and boy scout camps (158 patients), one was a municipal outbreak (an estimated few hundred patients), one occurred in a health resort (approx. 40 patients) and one was a family-and-friends outbreak (25 patients). The described outbreaks - except the municipal outbreak, which was thought to be waterborne - pointed clearly to the relevance of person-to-person transmission in the epidemiology of the NLVs. Furthermore, one outbreak in a school camp was prolonged and showed the importance of disinfection of contaminated environments (e.g. beds, toilets) after a NLV-outbreak to prevent further infections.

\section{Tasting Olive Oil - 'How Experts Do It and How Consumers Could Do It'}

\section{Annette Bongartz \\ University of Applied Sciences \\ Wädenswil}

Experts evaluate different qualitative criteria of olive oils with chemo-physical and organoleptical methods as priority. Guidelines from the EC and the IOOC (Int. Olive Oil Council) supply relevant parameters and values for the evaluation of the three different categories of olive oil 'Olive Oil', 'Virgin Olive Oil' and 'Extra Virgin Olive Oil'.

The latest example of a sensory assessment to evaluate the organoleptic criteria of 'Extra Virgin Olive Oil' was the ' 1 st. International Olive Oil Award - Zurich 2002' (http://www.oliveoilaward.ch).

To get an objective view of the organoleptical characteristics of olive oils either as an expert or a consumer - it is necessary to consider the following points:

Firstly it is important how olive oils are presented for tasting. Dark-coloured glasses are ideal, with a wide belly and a narrow neck, to displace the effect of the colour and to make sure that the aromatic compounds can develop but cannot escape. Secondly knowledge of the different organoleptical criteria - positive (mild, medium and intensive fruitiness) and negative (different offflavours) - that can occur in olive oils, is es- sential for each tester. And last but not least it is important to make sure that the correct sequence during tasting is adhered to:

- Presentation (if possible in special glasses: wide belly, narrow neck, dark blue)

- Swivelling of the olive oil in the glass (to develop the aromatic compounds)

- Smelling (with the nose, that is 'nasal' and 'retro-nasal')

- Evaluation of the aromatic impression

- Tasting (with the tongue)

- Evaluation of the taste impression

- Evaluation of the general harmonic impression

- Documentation of the results

- Neutralization after each oil (with sliced apples and water to get rid of the oilfilm)

\section{Phytochemicals: Focus on Bakery Products and Fruits}

\section{Beatrice Baumer \\ University of Applied Sciences \\ Wädenswil}

Phytochemicals have become increasingly significant in the last ten years. In contrast to primary plant stuffs (carbohydrates, proteins, fats, mineral salts, vitamins), they have no primary nutritional properties. They are produced by plants as pest and disease repellents, and also as growth regulators and colourings to attract insects and other animals. Phytochemicals are today considered to have an important role in nutrition and to be bioactive substances. They represent interesting components in the area of food absorption, with additional health benefits and occur in concentrations of up to $1 \%$ in bulbs, roots, leaves and fruit. On the basis of their chemical structure, phytochemicals can be divided into eleven groups with various effects.

The function of phytochemicals has been proven by different scientists in animal tests. Because of the large number of phytochemicals - Watzl and Leitzmann estimated in 1999 that there are about 100000 different phytochemicals - it is very difficult to find data on phytochemicals and their behaviour during food processing. The topic of the current research is therefore to investigate their behaviour and degradation during the production and storage of food.

Received: May 18, 2002 